\title{
Phenotypic and molecular characterization of quinolone resistance in Mycobacterium abscessus subsp. bolletii recovered from postsurgical infections
}

\begin{abstract}
Correspondence
Rafael Silva Duarte

rsduarte@ufrj.br
\end{abstract}

Received 9 June 2011

Accepted 6 September 2011
Vinicius Calado Nogueira de Moura, ${ }^{1}$ Marlei Gomes da Silva, ${ }^{2}$ Karen Machado Gomes, ${ }^{2}$ Fábrice Santana Coelho, ${ }^{3}$ Jorge Luiz Mello Sampaio, ${ }^{4}$ Fernanda Carvalho de Queiroz Mello, ${ }^{5}$ Maria Cristina da Silva Lourenço, ${ }^{6}$ Efigênia de Lourdes Teixeira Amorim ${ }^{7}$ and Rafael Silva Duarte ${ }^{2}$

\footnotetext{
${ }^{1}$ Departamento de Microbiologia, Imunologia e Parasitologia, Faculdade de Ciências Médicas, Universidade do Estado do Rio de Janeiro, Rio de Janeiro, Brazil

${ }^{2}$ Instituto de Microbiologia, Universidade Federal do Rio de Janeiro, Rio de Janeiro, Brazil

${ }^{3}$ Hospital Universitário Pedro Ernesto, Rio de Janeiro, Brazil

${ }^{4}$ Instituto Fleury de Ensino e Pesquisa, São Paulo, Brazil

${ }^{5}$ Instituto de Doenças do Tórax, Rio de Janeiro, Brazil

${ }^{6}$ Instituto de Pesquisa Evandro Chagas, Fundação Oswaldo Cruz, Rio de Janeiro, Brazil

${ }^{7}$ Sérgio Franco Medicina Diagnóstica, Rio de Janeiro, Brazil
}

\begin{abstract}
Several outbreaks of infections caused by rapidly growing mycobacteria (RGM) were reported in many Brazilian states (2032 notified cases) from 2004 to 2010. Most of the confirmed cases were mainly associated with Mycobacterium massiliense (recently renamed as Mycobacterium abscessus subsp. bolletii) BRA100 clone, recovered from patients who had undergone invasive procedures in which medical instruments had not been properly sterilized and/or disinfected. Since quinolones have been an option for the treatment of general RGM infections and have been suggested for therapeutic schemes for these outbreaks, we evaluated the in vitro activities of all generations of quinolones for clinical and reference RGM by broth microdilution, and analysed the peptide sequences of the quinolone resistance determining regions (QRDRs) of GyrA and GyrB after DNA sequencing followed by amino acid translation. Fifty-four isolates of $M$. abscessus subsp. bolletii, including clone BRA100, recovered in different states of Brazil, and 19 reference strains of RGM species were characterized. All 54 M. abscessus subsp. bolletii isolates were resistant to all generations of quinolones and showed the same amino acids in the QRDRs, including the Ala-83 in GyrA, and Arg-447 and Asp-464 in GyrB, described as being responsible for an intrinsic low level of resistance to quinolones in mycobacteria. However, other RGM species showed distinct susceptibilities to this class of antimicrobials and patterns of mutations contrary to what has been traditionally defined, suggesting that other mechanisms of resistance, different from gyrA or gyrB mutations, may also be involved in resistance to high levels of quinolones.
\end{abstract}

\section{INTRODUCTION}

Rapidly growing mycobacteria (RGM) are common in the natural environment, especially in water sources, and may contaminate medical solutions and equipment (BrownElliott \& Wallace, 2002). RGM have emerged as important human pathogens, and have been responsible for an
Abbreviations: QRDR, quinolone resistance determining region; RGM, rapidly growing mycobacteria.

The accession numbers for the sequences determined in this study are GU831598, GU831599, HQ285728, HQ285729-HQ285760 and HO324096-HQ324105. 
increasing number of health-care-associated infections (Brown-Elliott \& Wallace, 2002; Cardoso et al., 2008; De Groote \& Huitt, 2006; Duarte et al., 2009; Phillips \& von Reyn, 2001; Viana-Niero et al., 2008; Wallace et al., 1998).

The species Mycobacterium massiliense was first described in 2004 (Adékambi et al., 2004) and it has been responsible for several outbreaks of wound infections related to medical procedures in many states of Brazil (Cardoso et al., 2008; Duarte et al., 2009; Viana-Niero et al., 2008). A taxonomic note has been recently published by Leão $\mathrm{et} \mathrm{al}$. (2011) reclassifying Mycobacterium massiliense and Mycobacterium bolletii as belonging to the same subspecies of $M$. abscessus, namely Mycobacterium abscessus subsp. bolletii as suggested by Sampaio (2010). This taxonomic note also reclassifies the previous Mycobacterium abscessus as $M$. abscessus subsp. abscessus.

The largest epidemiological event associated with RGM in Brazil occurred between 2006 and 2007, when 1051 notified possible cases of postsurgical infections were spread around 63 hospitals in the state of Rio de Janeiro. During this epidemic, isolates belonging to a single clone of $M$. abscessus subsp. bolletii (previously named M. massiliense), designated BRA100, showed highly similar patterns by PFGE to those recovered in previous outbreaks in a few Brazilian states (Duarte et al., 2009). Randomly selected isolates showed resistance to ciprofloxacin (a second-generation fluoroquinolone), cefoxitin and doxycycline (Duarte et al., 2009). Futhermore, moxifloxacin (a fourth-generation fluoroquinolone with strong activity against Mycobacterium tuberculosis) was strongly suggested as a potential substitute for therapeutic schemes to treat $M$. abscessus subsp. bolletii infections, although not evaluated before.

The quinolones represent an option for antimicrobial treatment and are active against RGM, especially against the Mycobacterium fortuitum group (Brown-Elliott \& Wallace, 2002). Fourth-generation quinolones are important drugs used in ophthalmology and are more effective against Gram-positive bacteria compared to the other generations (Scoper, 2008; Stroman et al., 2005). This generation has also been demonstrated to be more active against mycobacteria and less affected by mutations in DNA gyrase (Brown-Elliott et al., 2002). Previous studies have demonstrated the importance of susceptibility testing for this generation of quinolones (Brown-Elliott et al., 2002; Höfling-Lima et al., 2005; Reddy et al., 2010).

Mycobacteria are naturally less susceptible to quinolones than other bacteria such as Escherichia coli (Wolfson \& Hooper, 1985), but the level of susceptibility to these drugs may differ considerably according to the mycobacterial species (Guillemin et al., 1995, 1998; Leysen et al., 1989; Li et al., 2004; Yew et al., 1994). Since topoisomerase IV has not been identified in the Mycobacterium tuberculosis genome (Cole et al., 1998), it is assumed that DNA gyrase is the only target for the quinolones in mycobacteria. The interaction between DNA gyrase and the quinolones involves conserved regions known as quinolone resistance determining regions (QRDRs) of the gyrA and gyrB genes (Yoshida et al., 1990, 1991).

In the E. coli numbering system, amino acids at positions 83 and 87 (90 and 94 in the M. tuberculosis numbering system) in the A subunit and at positions 426,447 and 464 (495, 516 and 533 in the M. tuberculosis numbering system) in the B subunit of DNA gyrase are frequently substituted in strains with acquired resistance to quinolones in mycobacteria (Cambau et al., 1994; Von Groll et al., 2009; Guillemin et al., 1995, 1998; Revel et al., 1994; Takiff et al., 1994; Wang et al., 2007) and seem to play a key role in the drug-enzyme interaction. Preliminary studies reported intrinsic resistance to quinolones in mycobacterial species due to the presence of the residues Ala- 83 in GyrA, and Arg-447 and Asn-464 in GyrB (Guillemin et al., 1998), which differ from the residues Ser-83 in GyrA, and Lys-447 and Ser-464 in GyrB, found in more susceptible species such as E. coli (Yoshida et al., 1990, 1991).

Due to the lack of data on the susceptibility of M. abscessus subsp. bolletii and some other RGM species to drugs, the aims of this study were to perform the phenotypic and molecular characterization of quinolone susceptibility of clinical strains of $M$. abscessus subsp. bolletii and other RGM species by determining the MIC of six quinolones (comprising all quinolone generations), including moxifloxacin, and to correlate it with amino acid variations in the QRDRs of GyrA and GyrB.

\section{METHODS}

Strains and growth conditions. The bacteria studied comprised 52 randomly selected clinical isolates of $M$. abscessus subsp. bolletii recovered from surgically infected sites in the states of Rio de Janeiro (48), São Paulo (1) and Goiás (3) obtained from clinical laboratories between 2004 and 2008; two epidemiologically unrelated isolates of $M$. abscessus subsp. bolletii isolated from sputum in the city of Rio de Janeiro; and 19 reference strains of RGM, namely M. abscessus subsp. abscessus ATCC 19977, M. abscessus subsp. bolletii CCUG 48898, Mycobacterium agri ATCC 27406, Mycobacterium aichiense ATCC 27280, Mycobacterium aurum ATCC 23366, Mycobacterium chelonae ATCC 14472, M. chelonae ATCC 35752, Mycobacterium chitae ATCC 19627, Mycobacterium chubuense ATCC 27278, Mycobacterium flavescens ATCC 14474, Mycobacterium neoaurum ATCC 25795, Mycobacterium fortuitum ATCC 6841, Mycobacterium gadium ATCC 27726, Mycobacterium parafortuitum ATCC 19686, Mycobacterium phlei ATCC 11758, Mycobacterium porcinum ATCC 33776, Mycobacterium rhodesiae ATCC 27024, Mycobacterium smegmatis ATCC 14468 and Mycobacterium vaccae ATCC 15783 . The clinical isolates of $M$. abscessus subsp. bolletii were selected and identified by PRA- $h s p 65$ and $r p o B$ sequencing, and typed by PFGE as previously described (Duarte et al., 2009). For the performance of the following assays, the isolates were inoculated on Löwenstein-Jensen (LJ) or Mueller-Hinton agar and incubated at $36{ }^{\circ} \mathrm{C}\left( \pm 1{ }^{\circ} \mathrm{C}\right)$ for 5 to 7 days.

Quinolone susceptibility testing. The susceptibility of all isolates to six quinolones, nalidixic acid (first generation), ciprofloxacin, ofloxacin and levofloxacin (second generation), sparfloxacin (third generation) and moxifloxacin (fourth generation) (all from SigmaAldrich) was determined by broth microdilution as recommended by the Clinical Laboratory Standards Institutes (CLSI) (CLSI, 2011). 
Table 1. Interpretative criteria for MICs for Enterobacteriaceae, Staphylococcus spp. and Streptococcus pneumoniae (CLSI, 2010)

\begin{tabular}{|c|c|c|c|c|c|c|c|c|c|}
\hline \multirow{3}{*}{$\begin{array}{l}\text { Antimicrobial } \\
\text { agent }\end{array}$} & \multicolumn{9}{|c|}{$\operatorname{MIC}\left(\mu \mathrm{g} \mathrm{ml}^{-1}\right)$} \\
\hline & \multicolumn{3}{|c|}{ Enterobacteriaceae } & \multicolumn{3}{|c|}{ Staphylococcus spp. } & \multicolumn{3}{|c|}{ Streptococcus pneumoniae } \\
\hline & $S^{*}$ & I & $\mathbf{R}$ & $S$ & I & $\mathbf{R}$ & $S$ & I & $\mathbf{R}$ \\
\hline Nalidixic acid & $\leqslant 16$ & - & $\geqslant 32$ & - & - & - & - & - & - \\
\hline Ofloxacin & $\leqslant 2$ & 4 & $\geqslant 8$ & $\leqslant 1$ & 2 & $\geqslant 4$ & $\leqslant 2$ & 4 & $\geqslant 8$ \\
\hline Levofloxacin & $\leqslant 2$ & 4 & $\geqslant 8$ & $\leqslant 1$ & 2 & $\geqslant 4$ & $\leqslant 2$ & 4 & $\geqslant 8$ \\
\hline Sparfloxacin & - & - & - & $\leqslant 0.5$ & 1 & $\geqslant 2$ & $\leqslant 0.5$ & 1 & $\geqslant 2$ \\
\hline Moxifloxacin & - & - & - & $\leqslant 0.5$ & 1 & $\geqslant 2$ & $\leqslant 1$ & 2 & $\geqslant 4$ \\
\hline
\end{tabular}

${ }^{\star}$ S, susceptible; I, intermediate; R, resistant.

Escherichia coli ATCC 25922 and Staphylococcus aureus ATCC 29213 were used as quality-control strains. The susceptibility patterns for ciprofloxacin and moxifloxacin were determined according to the MIC breakpoints defined by CLSI for RGM (CLSI, 2011). For the other quinolones the interpretative criteria for MIC were based on the breakpoints described for Enterobacteriaceae, Staphylococcus spp. and Streptococcus pneumoniae (Table 1, CLSI, 2010).

Sequencing of the QRDRs of the gyrA and gyrB genes. DNA was obtained from cell lysates by suspending a loopful of recent growth on LJ in sterile distilled water, followed by thermal lysis for $10 \mathrm{~min}$ and freezing at $-20{ }^{\circ} \mathrm{C}$. The PCR amplification of gyrA was performed as previously described (Guillemin et al., 1995) using Platinum Taq DNA polymerase (Invitrogen Life Technologies) and the primers Pri9 (5'-CGCCGCGTGCTSATGCRATG-3') and Pri8 (5'-YGGTGGRTCRTTRCCYGGCGA-3'). The amplification of gyrB was performed as previously described (Guillemin et al., 1995) using the same polymerase and the primers GyrbD ( $5^{\prime}$-CCGAYTGCCGTTCSACGGAT- $3^{\prime}$ ) and GyrbE (5'-CGGCCATCARCACGATCTTG-3').

The amplicons of $\operatorname{gyr} A(216 \mathrm{bp})$ and $\operatorname{gyrB}(268 \mathrm{bp})$ were purified with the Illustra GFX PCR DNA and Gel Band Purification kit (GE Healthcare). After purification, $5 \mathrm{ng}$ of each purified PCR product was sequenced by using an ABI PRISM 3700 DNA Analyser and a BigDye Terminator cycle sequencing kit (Applied Biosystems) (Otto et al., 2008).
Sequence analysis. The sequences obtained were analysed with BioEdit Sequence Alignment Editor version 7.0.5.1 and compared with those deposited in the GenBank database by using BLAST (http:// www.ncbi.nlm.nih.gov/BLAST). The phylogenetic tree was constructed using MEGA version 4.1. M. tuberculosis strain ATCC 25618 (H37Rv) was included as reference for sequence alignment and phylogenetic analysis.

\section{RESULTS}

\section{Quinolone susceptibility testing}

All 52 clinical isolates showed $>85 \%$ similarity among the patterns obtained by PFGE, and were named as belonging to $M$. abscessus subsp. bolletii BRA100 clone based on the criteria of Duarte et al. (2009). These isolates, as well as the two non-clonal ones (obtained from sputa) showed high but not identical MICs for all quinolones tested (range from 8 to $>2048 \mu \mathrm{g} \mathrm{ml}^{-1}$ ). The highest MICs were for nalidixic acid (Tables 2 and 3 ).

The quinolone susceptibility testing among reference strains showed variable susceptibility patterns. Considering the

Table 2. MIC patterns of six quinolones for $54 \mathrm{M}$. abscessus subsp. bolletii clinical isolates

\begin{tabular}{|c|c|c|c|c|}
\hline \multirow{2}{*}{$\begin{array}{l}\text { Antimicrobial } \\
\text { agent }\end{array}$} & \multicolumn{4}{|c|}{$\operatorname{MIC}\left(\mu \mathrm{g} \mathrm{ml}^{-1}\right)$} \\
\hline & Range & Mode $^{\star}$ & $\mathrm{MIC}_{50} \dagger$ & $\mathrm{MIC}_{90} \neq$ \\
\hline Nalidixic acid & - & $>2048$ & $>2048$ & $>2048$ \\
\hline Ofloxacin & $>128-32$ & 128 & 128 & $>128$ \\
\hline Ciprofloxacin & $>32-8$ & 16 & 16 & $>32$ \\
\hline Levofloxacin & $>128-16$ & 64 & 64 & $>128$ \\
\hline Sparfloxacin & $>128-32$ & 128 & 128 & $>128$ \\
\hline Moxifloxacin & $32-8$ & 16 & 16 & 32 \\
\hline
\end{tabular}

${ }^{*}$ The value that occurred most frequently among the isolates tested.

$\dagger$ The MIC capable of preventing the growth of $50 \%$ of the isolates.

¥The MIC capable of preventing the growth of $90 \%$ of the isolates. 
Table 3. Correlation between the MICs of quinolones against 19 reference strains of RGM and the amino acid residue at position 83 in the QRDR in the A subunit of DNA gyrase

\begin{tabular}{|c|c|c|c|c|c|c|c|c|}
\hline Species & Representative strain & \multicolumn{6}{|c|}{$\operatorname{MIC}\left(\mu \mathrm{g} \mathrm{ml}^{-1}\right)^{*}$} & $\begin{array}{c}\text { Amino acid } \\
83 \dagger \text { in GyrA } \\
\text { QRDR }\end{array}$ \\
\hline \multicolumn{9}{|l|}{ Susceptible $\ddagger$} \\
\hline M. aichiense & ATCC 27280 & 16 & 1 & $\leqslant 0.06$ & $\leqslant 0.25$ & $\leqslant 0.25$ & $\leqslant 0.25$ & Ser \\
\hline M. chitae & ATCC 19627 & 1024 & 2 & 0.5 & 8 & 0.5 & $\leqslant 0.5$ & Ser \\
\hline M. chubuense & ATCC 27278 & 4 & $\leqslant 0.25$ & $\leqslant 0.06$ & $\leqslant 0.25$ & $\leqslant 0.25$ & $\leqslant 0.5$ & Ser \\
\hline M. flavescens & ATCC 14474 & 2 & $\leqslant 0.25$ & 0.06 & $\leqslant 0.25$ & $\leqslant 0.25$ & $\leqslant 0.5$ & Ser \\
\hline M. fortuitum & ATCC 6841 & 64 & $\leqslant 0.25$ & $\leqslant 0.06$ & $\leqslant 0.25$ & $\leqslant 0.25$ & $\leqslant 0.5$ & Ser \\
\hline M. neoaurum & ATCC 25795 & 2 & $\leqslant 0.25$ & $\leqslant 0.06$ & $\leqslant 0.25$ & $\leqslant 0.25$ & $\leqslant 0,5$ & Ser \\
\hline M. parafortuitum & ATCC 19686 & 16 & $\leqslant 0.25$ & 0.06 & $\leqslant 0.25$ & $\leqslant 0.25$ & $\leqslant 0.5$ & Ser \\
\hline M. smegmatis & ATCC 14468 & 512 & 1 & 0.5 & $\leqslant 0.25$ & $\leqslant 0.25$ & $\leqslant 0.5$ & Ala \\
\hline M. vaccae & ATCC 15483 & 128 & 0.5 & 0.5 & $\leqslant 0.25$ & $\leqslant 0.25$ & $\leqslant 0.5$ & Ser \\
\hline \multicolumn{9}{|l|}{ Resistant $\ddagger$} \\
\hline $\begin{array}{l}\text { M. abscessus subsp. } \\
\text { abscessus }\end{array}$ & ATCC 19977 & $>2048$ & $>128$ & 16 & 128 & 128 & 16 & Ala \\
\hline $\begin{array}{l}\text { M. abscessus subsp. } \\
\text { bolletii }\end{array}$ & CCUG 48898 & $>2048$ & 128 & 16 & 32 & 128 & 16 & Ala \\
\hline M. chelonae & ATCC 14472 & 512 & 32 & 16 & 16 & 8 & 2 & Ala \\
\hline M. gadium & ATCC 27726 & $>2048$ & 64 & 32 & 64 & 64 & 16 & Ser \\
\hline M. agri & ATCC 27406 & $>2048$ & 32 & $>32$ & 64 & 64 & 4 & Ser \\
\hline
\end{tabular}

${ }^{\star}$ NAL, nalidixic acid; OFX, ofloxacin; CIP, ciprofloxacin; LVX, levofloxacin; SPX, sparfloxacin; MOX, moxifloxacin.

$\nmid$ Numbering system used for E. coli.

$\ddagger$ Based on MIC breakpoints for ciprofloxacin.

MIC interpretations for ciprofloxacin, the RGM fell into two groups: susceptible and resistant (Table 3). The susceptible group comprised M. aichiense ATCC 27280, M. aurum ATCC 23366, M. chelonae ATCC 35752, M. chitae ATCC 19627, M. chubuense ATCC 27278, M. flavescens ATCC 14474, M. fortuitum ATCC 6841, M. gadium ATCC 27726, M. neoaurum ATCC 25795, M. parafortuitum ATCC 19686, M. phlei ATCC 11758, M. porcinum ATCC 33776, M. rhodesiae ATCC 27024), M. smegmatis ATCC 14468 and M. vaccae ATCC 15483. The resistant group comprised M. abscessus subsp. abscessus ATCC 19977, M. abscessus subsp. bolletii CCUG 48898, M. agri ATCC 27406 and M. chelonae ATCC 14472. The strain for which the MIC was highest was M. abscessus subsp. abscessus ATCC 19977, and the lowest MIC was for M. neoaurum ATCC 25725 (Table 3). The reference strain CCUG 48898 of M. abscessus subsp. bolletii showed resistance to high levels of all quinolones, similar to the clinical isolates.

\section{Nucleotide sequences of the gyrA and gyrB QRDRs}

The nucleotide sequences of $g y r A$ and $g y r B$ QRDR differed among the mycobacterial species (Fig. 1); however in gyrA they were identical for all clinical isolates of $M$. abscessus subsp. bolletii and the reference strain CCUG 48898. The homology analysis of the gyrA nucleotide sequences is shown in Fig. 2.

The nucleotide sequences of the gyrB QRDR of M. abscessus subsp. bolletii showed four different patterns. In pattern 1 (P1), consisting of 50 BRA100 isolates and the two nonclonal ones, sequences (GU831599) were identical and showed $95.7 \%$ homology to the CCUG 48898 strain (HQ324105). Sequences in pattern 2 (P2, HQ285758), consisting of two BRA100 isolates from Rio de Janeiro, were identical to the CCUG 48898 strain. Patterns 3 (P3, one BRA100 isolate from Rio de Janeiro, HQ285759) and 4 (P4, one BRA100 isolate isolated from Goiás, HQ285760) showed, respectively, 99.1 and $97.4 \%$ homology to the CCUG 48898 strain (Fig. 3).

\section{Peptide sequences of the GyrA and GyrB QRDRs}

The peptide sequences of the GyrA and GyrB QRDRs obtained for RGM were aligned with the peptide sequences of M. tuberculosis, E. coli and Staphylococcus aureus, using 
作

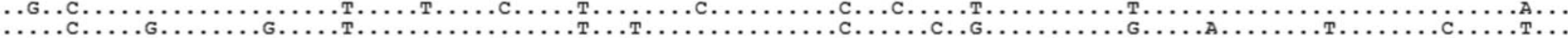

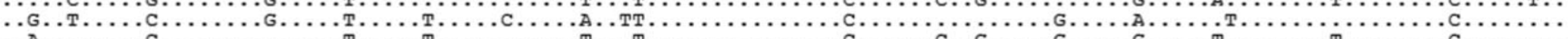

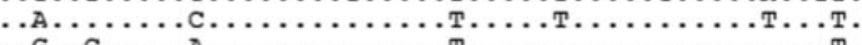
G...

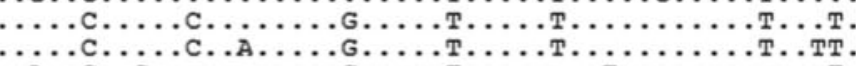

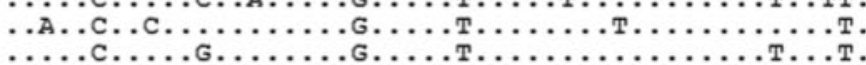

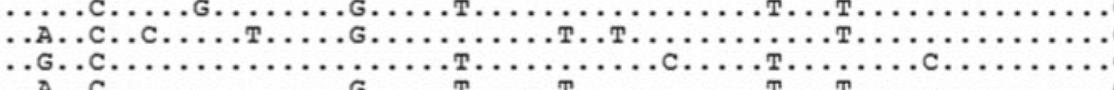

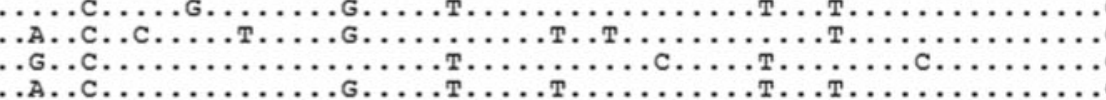
.А. С. . . G..c.

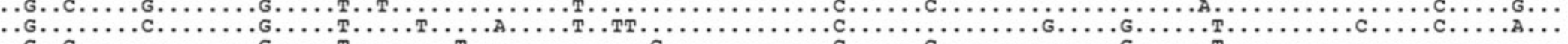

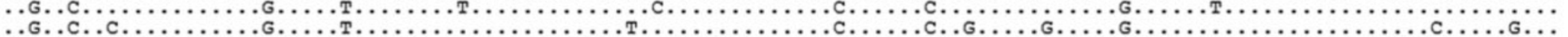

\section{QRDR gyrB}

1414

M. tuberculosis M. abscessus

M. agri

M. aichiense

M. aurum

M. chelonae 1

M. chelonae 2

M. chitae

M. chubuense
M. flavescens

M. fortuitum

M. gadium

I'ACTCGGCCGGCGGTTCTGCAAAAAGCGGTCGCGATTCGATGTTCCAGGCGATACTTCCGCTGCGCGGCAAGATCATCAATGTGGAGAAAGCGCGCATCGACCGGGTGCTAAAGAAC с....

M. massiliense $P$ 1

M. massiliense P2

M. massiliense P3

M. massiliense
M. neoaurum

M. parafortuitum

M. phlei

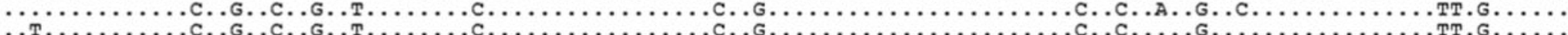
т.

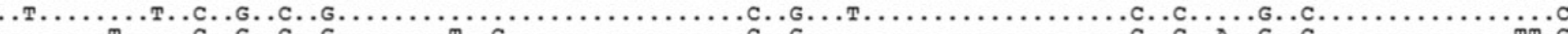

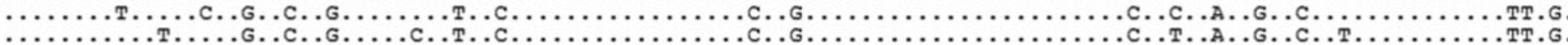
.

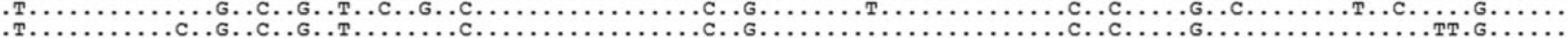
т...

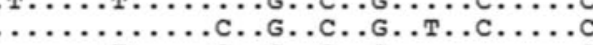

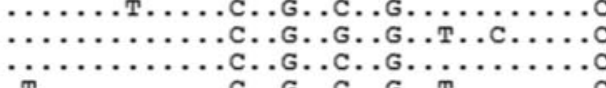

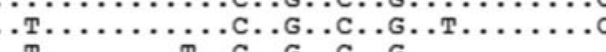

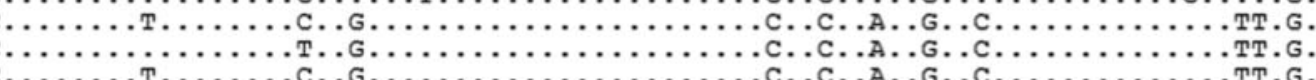

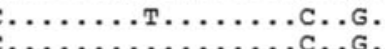
C.C.A.G.

Fig. 1. Alignment of the nucleotide sequences of the QRDRs of gyrA and gyrB from species of RGM. Sequences extend from nucleotides 220 to 339 for $g y r A$ and from nucleotides 1414 to 1530 for $g y r B$, in the numbering system used for $M$. tuberculosis. Sequences of $M$. tuberculosis were used as the reference and dots represent identical nucleotides. M. chelonae 1 represents ATCC 35752, M. chelonae 2 represents ATCC 14472; M. abscessus has been recently renamed as M. abscessus subsp. abscessus, and M. massiliense as $M$. abscessus subsp. bolletii. 


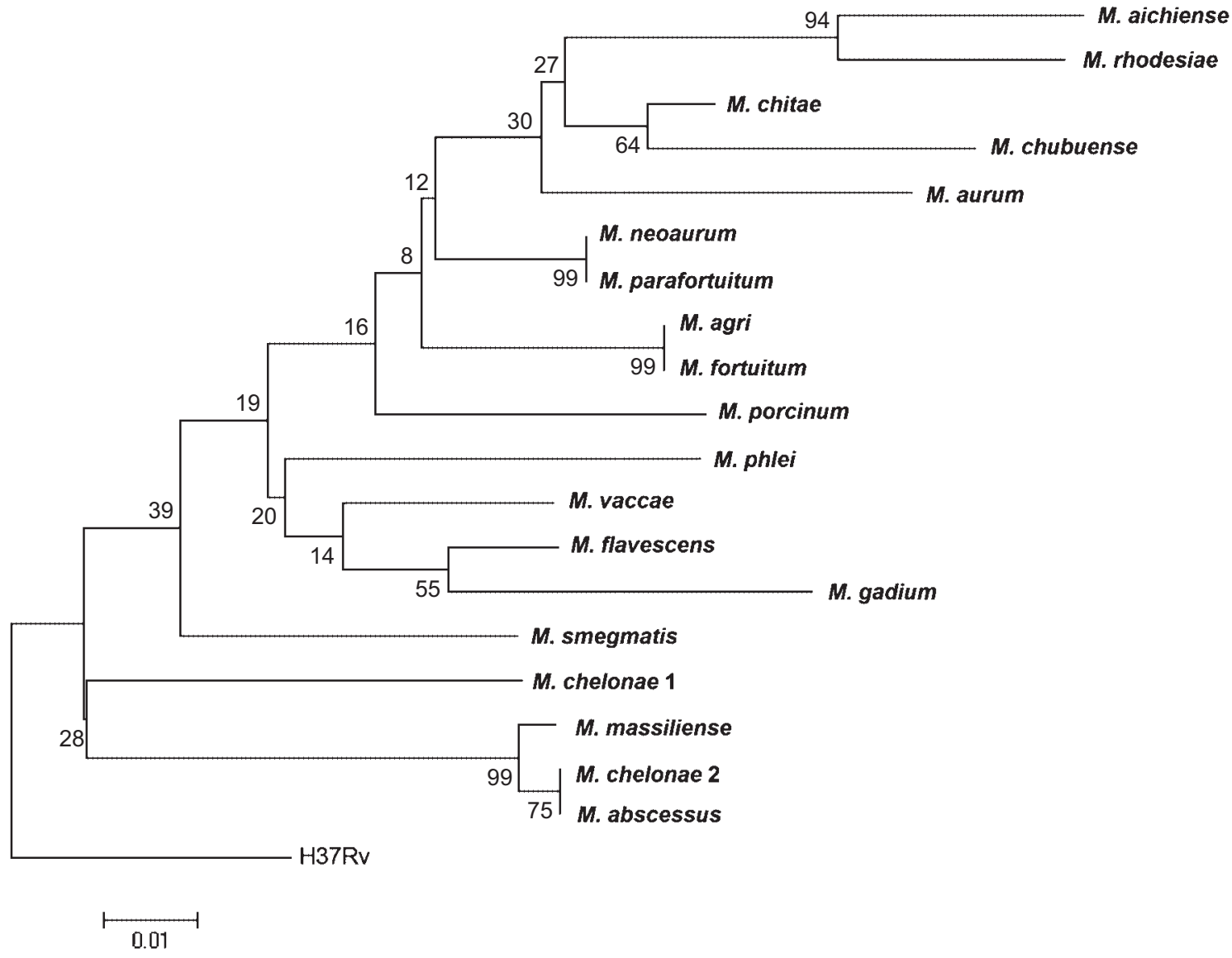

Fig. 2. Phylogenetic tree constructed by the neighbour-joining method using the nucleotide sequence of the $g y r A$ QRDR. $M$. chelonae 1 represents ATCC 35752, M. chelonae 2 represents ATCC 14472; $M$. abscessus has been recently renamed as $M$. abscessus subsp. abscessus, and $M$. massiliense as $M$. abscessus subsp. bolletii. The numbers at the nodes are bootstrap values from 1000 resamplings.

the numbering system of the DNA gyrase of E. coli (Fig. 4). In all RGM strains, the peptide sequences were identical for the GyrB QRDR and showed Arg-447 and Asn-464, the same residues as found in Staphylococcus aureus and Streptococcus pneumoniae.

The peptide sequences of the GyrA QRDR were identical for all isolates of M. abscessus subsp. bolletii and showed Ala-83, also found in Staphylococcus aureus (Ito et al., 1994) and Streptococcus pneumoniae (Pan et al., 1996). Except for the Ser-83 $\rightarrow$ Ala- 83 variation, the peptide sequence of GyrA was identical for all RGM strains. Species that showed Ser-83 were M. agri ATCC 27406, M. aichiense ATCC 27280, M. chubuense ATCC 27278, M. aurum ATCC 23366, M. chelonae ATCC 35752, M. chitae ATCC 19627, M. flavescens ATCC 14474, M. fortuitum ATCC 6841, M. gadium ATCC 27726, M. neoaurum ATCC 25795, M. parafortuitum ATCC 19686, M. porcinum ATCC 33776, M. rhodesiae ATCC 27024 and M. vaccae ATCC 15483. Species that showed Ala-83 were M. abscessus subsp. abscessus ATCC 19977, M. chelonae ATCC 14472, M. abscessus subsp. bolletii CCUG 48898, M. phlei ATCC 11758 and M. smegmatis ATCC 14468.
The association between the MIC results and amino acid 83 in GyrA (Table 3) shows that the presence of Ser-83 and Ala-83 corresponded respectively to the susceptible and resistant profiles for most species. However, exceptions to this were observed in M. phlei and M. smegmatis strains, which contained Ala-83 and were classified as susceptible, and also in M. agri and M. gadium, which contained Ser-83 and were resistant to quinolones.

\section{DISCUSSION}

Over the past few years, RGM have played an important role as emerging health-care-associated pathogens (BrownElliott \& Wallace, 2002; De Groote \& Huitt, 2006; Phillips \& von Reyn, 2001; Wallace et al., 1998). An epidemic such as the one that occurred recently in different states of Brazil (see Introduction), had never been described before worldwide and it has been considered as an epidemiological emergency. Since RGM have been rising as a significant health problem, it is essential to evaluate their susceptibility patterns to current antimycobacterial drugs 


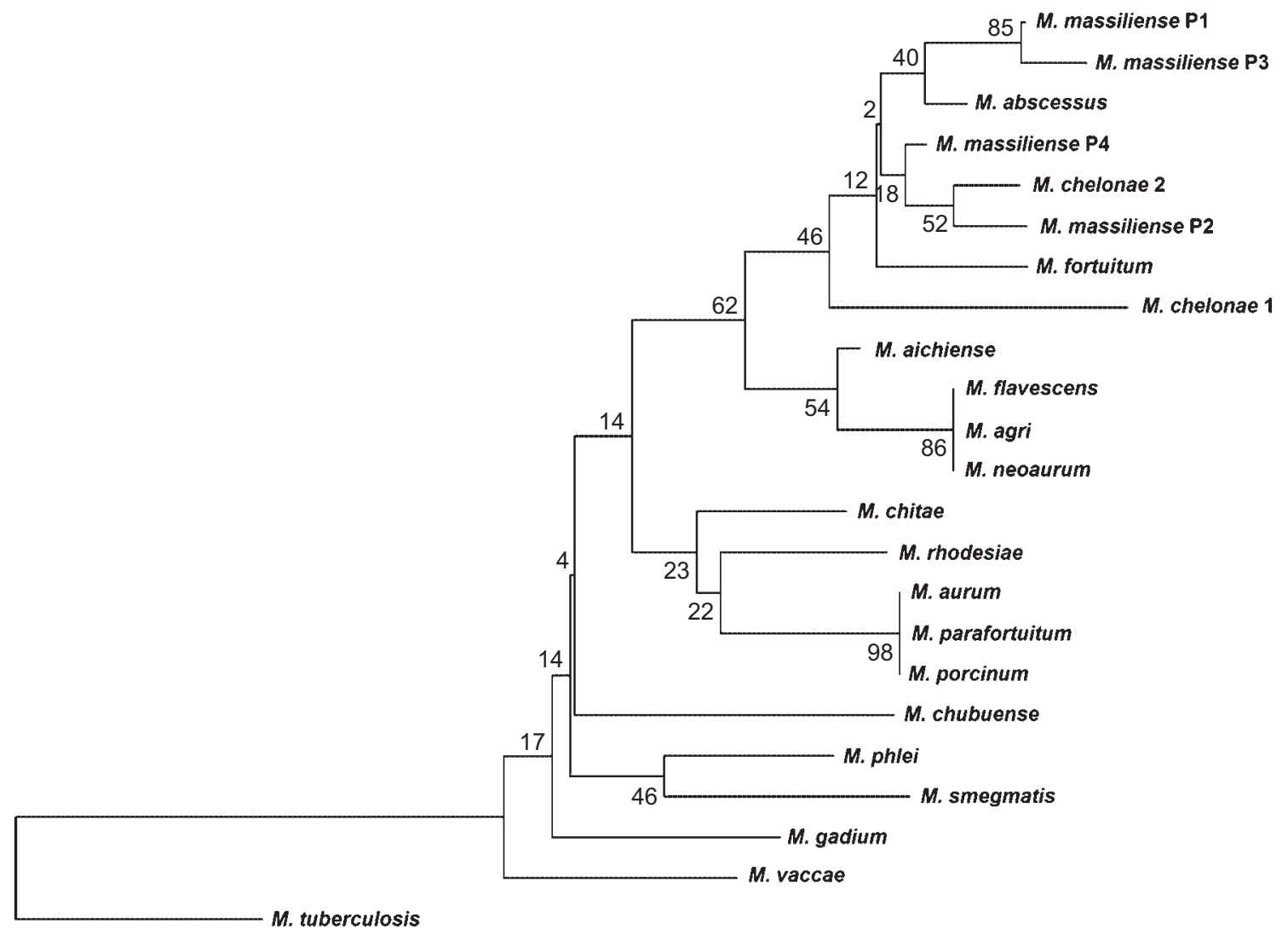

Fig. 3. Phylogenetic tree constructed by the neighbour-joining method using the nucleotide sequence of $g y r B$ QRDR. $M$. chelonae 1 represents ATCC 35752, M. chelonae 2 represents ATCC 14472; $M$. abscessus has been recently renamed as $M$. abscessus subsp. abscessus, and $M$. massiliense as $M$. abscessus subsp. bolletii. The numbers at the nodes are bootstrap values from 1000 resamplings.

and to describe mechanisms of resistance to antimicrobial agents in order to improve treatment and drug choice.

In a previous study (Duarte et al., 2009) resistance to ciprofloxacin was observed in some $M$. abscessus subsp. bolletii isolates recovered from postsurgical infections; however, the susceptibility of these strains to quinolones of other generations remained unknown until this present study. In the present study, most of the epidemic isolates were recovered from all hospitals with confirmed cases in the state of Rio de Janeiro, accounting for around $32 \%$ of the total of isolates obtained $(n=148)$, and additional representative strains from another two states with cases confirmed. During the characterization, these isolates exhibited clonal clustering by PFGE and were named as clone BRA100 (Duarte et al., 2009; Leão et al., 2010). Knowledge about potential emergence of M. abscessus subsp. bolletii and its antimicrobial resistance profile may contribute to systematic recovery of new isolates from different clinical sources and regions and improvement of their characterization.

The MIC results for nalidixic acid, ofloxacin, ciprofloxacin, levofloxacin, sparfloxacin and moxifloxacin demonstrated that quinolones of all generations were not inhibitory against any isolate of $M$. abscessus subsp. bolletii, including non-BRA100 isolates. The MIC results were similar to those found in previous studies (Guillemin et al., 1995, 1998), and previously unknown quinolone susceptibility patterns of other reference strains were also characterized in the present study. Quinolones are widely used in Brazil for treatment of urinary tract, respiratory, gall bladder, skin and gonococcal infections, and also used prophylactically for urinary or general surgery. Additionally, moxifloxacin has been described as a possible alternative for treatment of tuberculosis, and Mycobacterium avium and Bacteroides fragilis infections. Incorrect prescription of these drugs in routine medical practice and their prophylactic use for gallbladder surgery may occur. No data on prescriptions of quinolones for these purposes were available in this study.

It is important to mention that the CLSI have breakpoints for just two quinolones, ciprofloxacin and moxifloxacin, and the recommendations have been described only for $M$. fortuitum group ( $M$. fortuitum, M. peregrinum and $M$. fortuitum third biovariant complex), M. chelonae, $M$. abscessus subsp. abscessus, M. mucogenicum and M. smegmatis group (M. smegmatis, M. goodi and M. wolinskyi). It was shown in this work that the breakpoints for the other 


\section{QRDR GyrA}

M. tuberculosis
M. abscessus
M. agri
M. aichiense
M. aurum
M. chelonae 1
M. chelonae 2
M. chitae
M. chubuense
M. flavescens
M. fortuitum
M. gadium
M. massiliense
M. neoaurum
M. parafortuitum
M. phlei
M. porcinum
M. rhodesiae
M. smegmatis
M. vaccae
E. coli
S. aureus
. pneumia

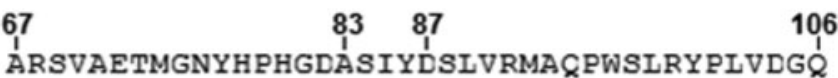

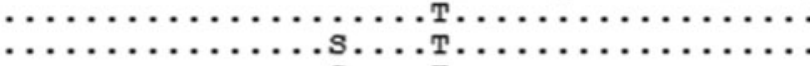

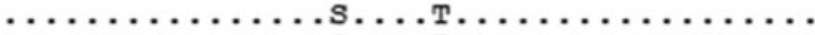

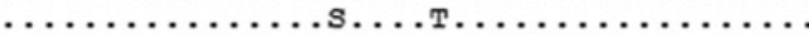

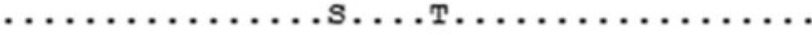

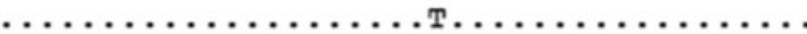

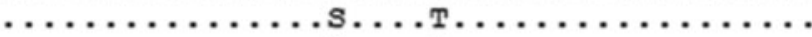

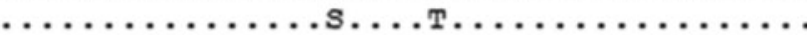

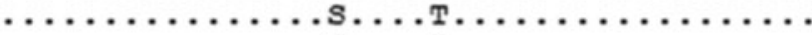

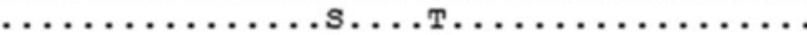

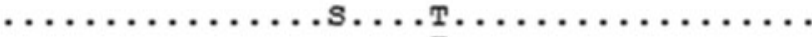

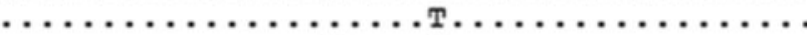

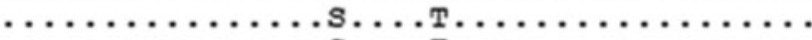

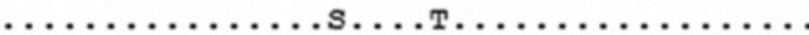

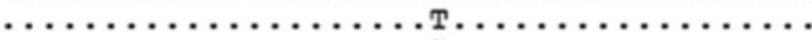

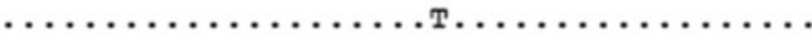

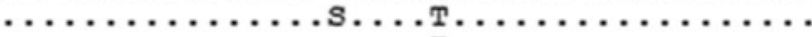

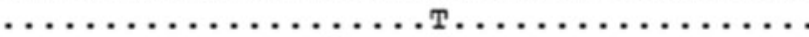

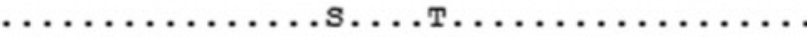
.V.GDVI.K..... SAV . TI...... . . . . . .

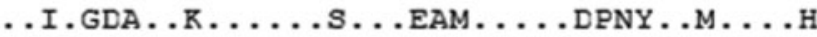
..ITGDV..K......... EAM..... . . . . ...H

\section{QRDR GyrB}

M. tuberculosis
M. abscessus
M. agri
M. aichiense
M. aurum
M. chelonae 1
M. chelonae 2
M. chitae
M. chubuense
M. flavescens
M. fortuitum
M. gadium
M. massiliense $\mathrm{P} 1$
M. massiliense $\mathrm{P} 2$
M. massiliense $\mathrm{P} 3$
M. massiliense P4
M. neoaurum
M. parafortuitum
M.phlei
M.porcinum
M. rhodesiae
M. smegmatis
M. vaccae
E. coli
S. aureus
S. pneumoniae

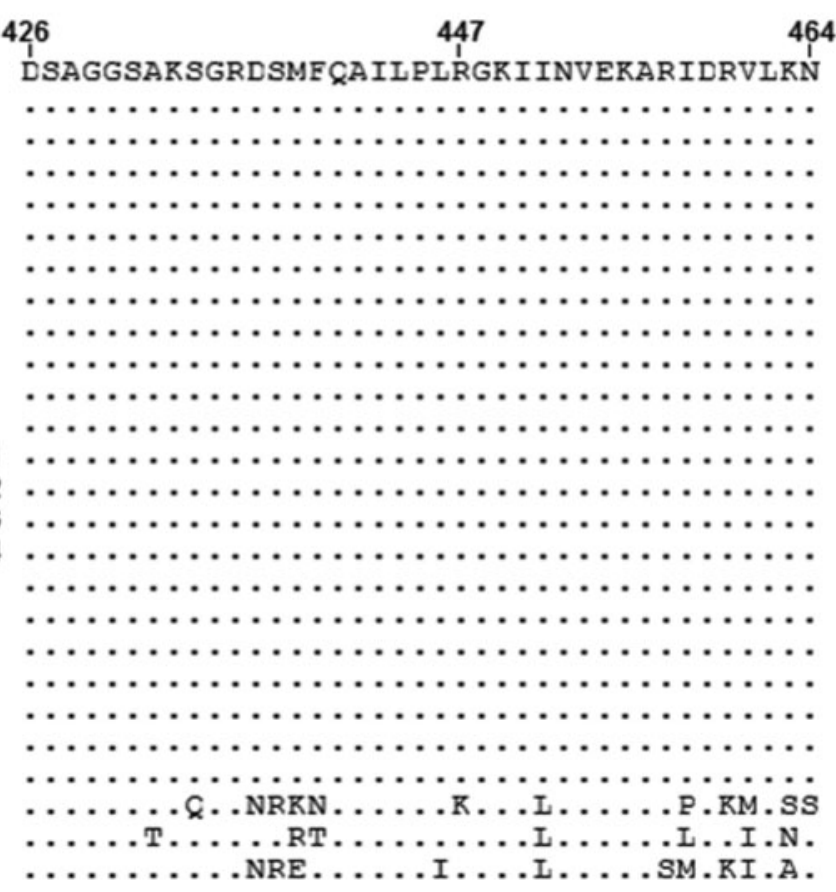

Fig. 4. Alignment of the peptide sequences of the QRDRs of GyrA and GyrB from species of RGM and from E. coli, Staphylococcus aureus and Streptococcus pneumoniae. Dots represent amino acids identical to those in M. tuberculosis. The GyrA QRDR extends from amino acid residues 67 to 106 , and the GyrB QRDR extends from amino acid residues 426 to 464 , in the numbering system used for $E$. coli. M. chelonae 1 represents ATCC 35752, M. chelonae 2 represents ATCC 14472; M. abscessus has been recently renamed as $M$. abscessus subsp. abscessus, and M. massiliense as M. abscessus subsp. bolletii. quinolones described in the CLSI M100-S20 document for E. coli, Staphylococcus aureus and Streptococcus pneumoniae (CLSI, 2010) were useful for determining the susceptibility pattern in mycobacterial species when compared to molecular results, suggesting a good applicability of these criteria to RGM. Since the previous CLSI M-24-A document for antimicrobial susceptibility testing in mycobacteria, published in 2003, new antimicrobial agents, including new generations of quinolones and additional mycobacterial species have been reported up to the newest version, published in 2011 (CLSI, 2011). So there is a need for continuous updating of this document, and information obtained in this study may be useful. In our study the susceptibility patterns for all generations of quinolones were precisely determined and reproducible for all RGM evaluated by using cation-adjusted Mueller-Hinton broth as recommended by CLSI.

The homology analysis demonstrated the high similarity between M. abscessus subsp. bolletii and M. abscessus subsp. 
abscessus for both gyrA and gyrB, but the RGM strains showed a different pattern for each tree. It is important to note that in $g y r A$, the sequences for clonal and non-clonal isolates were identical, whereas in $g y r B$, the clonal isolates had four different patterns (P1, P2, P3 and P4), even when comparing only the isolates belonging to the BRA100 clone. P2 was identical to the reference sequence of $M$. abscessus subsp. bolletii CCUG 48898, which may represent an intra- or inter-clone divergence between isolates. Possible reasons for this diversity being detected only in gyrB sequences are (i) different isolates from the same clone have suffered different evolutionary pressures, (ii) gyrB might play a different and possible minor role in the susceptibility of this species to quinolones or (iii) it represents a naturally hypervariable region of RGM genomes. Additionally, these results suggest that similar PFGE patterns of epidemiologically related isolates (BRA100, $>85 \%$ similarity) do not represent identical genome sequences, and even different phenotypic profiles may be observed in following studies evaluating distinct biological characteristics. Thus the concept of defining clonal strains for $M$. abscessus subsp. bolletii based only on PFGE patterns may be assumed with restrictions.

It was also observed that M. chelonae ATCC 14472 showed $100 \%(g y r A)$ and $99 \%(g y r B)$ homology to M. abscessus subsp. bolletii CCUG 50184 by using BLAST analysis. Furthermore, the $r p o B$ sequence of this strain showed high similarity to M. abscessus subsp. bolletii (unpublished data). These results may suggest that this strain is in fact $M$. abscessus subsp. bolletii and not M. chelonae.

Considering the peptide sequences of GyrA, variation in residue 83 was found in the QRDR in the RGM species. Less susceptible species of RGM studied in this work, including M. abscessus subsp. abscessus and M. abscessus subsp. bolletii, had Ala instead of Ser in this position, the same residues as found in less susceptible species such as Staphylococcus aureus (Ito et al., 1994) and Streptococcus pneumoniae (Pan et al., 1996). However, contrary to what has been traditionally described, $M$. agri and $M$. gadium were clear exceptions, since they were resistant to quinolones yet had Ser at this locus. This suggests that the variation between Ala and Ser in residue 83 may not be the unique determinant for a resistant or susceptible phenotype. The opposite was also observed and supports this hypothesis: strains phenotypically susceptible to quinolones, such as M. phlei and M. smegmatis, had Ala as residue 83 in the GyrA QRDR. Previous authors hypothesized that the differences in the structures between Ser and Ala should be related to intrinsic resistance in some species, such as M. abscessus subsp. bolletii. It is thought that Ala cannot provide a hydrogen bond because of its lack of a hydroxyl radical, providing the resistant profile (Guillemin et al., 1995), but this does not explain the variations observed in the present study.

The peptide sequences of QRDR of GyrB were identical in all RGM tested and contained the residues Arg-447 and
Asn-464, which also seem to be associated with lesser susceptibility to quinolones, compared to other bacteria. These residues differ from those found in E. coli (Lys-447 and Ser-464), a more susceptible species. Guillemin et al. (1998) argued that since Arg is bulkier than Lys and has an additional positive charge, and Asn is non-hydroxylated and bulkier than Ser, their presence could decrease the interaction between the DNA gyrase and the drug.

A recent study of gyrA and gyrB mutations in ciprofloxacin-resistant $M$. abscessus subsp. bolletii isolated from an outbreak in southern Brazil showed the substitution Ala$90 \rightarrow$ Val-90 (Ala-83 $\rightarrow$ Val-83 using the E. coli numbering system) in the QRDR of GyrA in $88.6 \%(31 / 35)$ of the isolates. This study also showed that $11.4 \%(4 / 35)$ of the isolates did not show this substitution and were resistant to ciprofloxacin. The isolates from this outbreak had a PFGE profile characteristic of the BRA100 clone (Monego et al., 2011). The BRA100 isolates studied in the present work did not show the substitution Ala-83 $\rightarrow$ Val-83 and they were also resistant to quinolones of all generations, not only to ciprofloxacin, suggesting that (i) the BRA100 clone as defined by PFGE criteria does not consist of isolates with the same genomic sequence, as previously discussed in our work; (ii) resistance to quinolones may involve not only mutations in QRDR of gyrA and gyrB but also other mechanisms of resistance.

Mechanisms of antimicrobial resistance besides the classic low cell wall permeability have already been described for mycobacteria and they involve porins (Danilchanka et al., 2008; Svetlíková et al., 2009; Stephan et al., 2004), efflux pumps (Li et al., 2004; Liu et al., 1996; Louw et al., 2009) and a pentapeptide $\mathrm{MfpA}$, which plays a role in quinolone resistance in M. smegmatis (Montero et al., 2001). It has been recently proposed that a single mechanism which confers a low level of resistance to quinolones should not be underestimated and the combination of several mechanisms could explain the level of resistance achieved in clinical isolates (Hernández et al., 2011). This might be the explanation for the high levels of resistance observed in M. massiliense and related RGM. Those mechanisms should also be studied and associated with the MIC results in order to complete the characterization of quinolone resistance.

In conclusion, even though quinolones of different generations may be effective against some species of RGM, they should not be used to treat M. abscessus subsp. bolletii infections due to the resistance to high levels found in this subspecies. The variation between Ser and Ala at position 83 may play a role in reduced susceptibility to quinolones in mycobacteria. However, this does not explain the high levels of resistance to all generations of quinolones in $M$. abscessus subsp. bolletii and related species, since the presence of Ala-83 in the GyrA QRDR is expected in some species of mycobacteria, including species susceptible to ciprofloxacin (Guillemin et al., 1995, 1998), and no other amino acid change was observed in both 
genes. Other mechanisms might be present in those species to explain such resistance. The present study represents an important aid to the knowledge about mechanisms of quinolone resistance in RGM and for future review of susceptibility criteria.

\section{ACKNOWLEDGEMENTS}

The Plataform-DNA Sequencing (PDTIS/FIOCRUZ), Rio de Janeiro, $\mathrm{RJ}$, Brazil, is acknowledged for performing the $r p o B, \operatorname{gyr} A$ and $g y r B$ sequencing procedures. We also thank Dr S. C. Leão for genomic analysis, and appreciated the collaboration of Alcon Laboratórios do Brasil Ltda by moxifloxacin donation. This study was supported in part by the Fundação de Amparo à Pesquisa do Estado do Rio de Janeiro (FAPERJ, projects no. 110.347/2007, 18123/2008, 111.497/ 2008 and 110.272/2010), the Conselho Nacional de Desenvolvimento Científico e Tecnológico ( $\mathrm{MCT} / \mathrm{CNPq}$, Edital Universal, projects no. 567037/2008-8, 473143/2007-0 and 473444/2010-0) and PDTIS FIOCRUZ

\section{REFERENCES}

Adékambi, T., Reynaud-Gaubert, M., Greub, G., Gevaudan, M. J., La Scola, B., Raoult, D. \& Drancourt, M. (2004). Amoebal coculture of "Mycobacterium massiliense" sp. nov. from the sputum of a patient with hemoptoic pneumonia. J Clin Microbiol 42, 5493-5501.

Brown-Elliott, B. A. \& Wallace, R. J., Jr (2002). Clinical and taxonomic status of pathogenic nonpigmented or late-pigmenting rapidly growing mycobacteria. Clin Microbiol Rev 15, 716-746.

Brown-Elliott, B. A., Wallace, R. J., Jr, Crist, C. J., Mann, L. \& Wilson, R. W. (2002). Comparison of in vitro activities of gatifloxacin and ciprofloxacin against four taxa of rapidly growing mycobacteria. Antimicrob Agents Chemother 46, 3283-3285.

Cambau, E., Sougakoff, W., Besson, M., Truffot-Pernot, C., Grosset, J. \& Jarlier, V. (1994). Selection of a gyrA mutant of Mycobacterium tuberculosis resistant to fluoroquinolones during treatment with ofloxacin. J Infect Dis 170, 479-483.

Cardoso, A. M., Martins de Sousa, E., Viana-Niero, C., Bonfim de Bortoli, F., Pereira das Neves, Z. C., Leão, S. C., Junqueira-Kipnis, A. P. \& Kipnis, A. (2008). Emergence of nosocomial Mycobacterium massiliense infection in Goiás, Brazil. Microbes Infect 10, 1552-1557.

CLSI (2010). Performance Standards for Antimicrobial Susceptibility Testing, 20th informational supplement. CLSI document M100-S20. Wayne, PA: Clinical Laboratory and Standards Institute.

CLSI (2011). Susceptibility Testing of Mycobacteria, Nocardiae, and other Aerobic Actinomycetes, approved standard, 2nd edn. CLSI document M24-A2. Wayne, PA: Clinical Laboratory and Standards Institute.

Cole, S. T., Brosch, R., Parkhill, J., Garnier, T., Churcher, C., Harris, D., Gordon, S. V., Eiglmeier, K., Gas, S. \& other authors (1998). Deciphering the biology of Mycobacterium tuberculosis from the complete genome sequence. Nature 393, 537-544.

Danilchanka, O., Pavlenok, M. \& Niederweis, M. (2008). Role of porins for uptake of antibiotics by Mycobacterium smegmatis. Antimicrob Agents Chemother 52, 3127-3134.

De Groote, M. A. \& Huitt, G. (2006). Infections due to rapidly growing mycobacteria. Clin Infect Dis 42, 1756-1763.

Duarte, R. S., Lourenço, M. C. S., Fonseca, L. S., Leão, S. C., Amorim, E. L. T., Rocha, I. L. L., Coelho, F. S., Viana-Niero, C., Gomes, K. M. \& other authors (2009). Epidemic of postsurgical infections caused by Mycobacterium massiliense. J Clin Microbiol 47, 2149-2155.
Guillemin, I., Cambau, E. \& Jarlier, V. (1995). Sequences of conserved region in the A subunit of DNA gyrase from nine species of the genus Mycobacterium: phylogenetic analysis and implication for intrinsic susceptibility to quinolones. Antimicrob Agents Chemother 39, 21452149.

Guillemin, I., Jarlier, V. \& Cambau, E. (1998). Correlation between quinolone susceptibility patterns and sequences in the $\mathrm{A}$ and $\mathrm{B}$ subunits of DNA gyrase in mycobacteria. Antimicrob Agents Chemother 42, 2084-2088.

Hernández, A., Sánchez, M. B. \& Martínez, J. L. (2011). Quinolone resistance: much more than predicted. Front Microbiol 2, 1-6.

Höfling-Lima, A. L., de Freitas, D., Sampaio, J. L. M., Leão, S. C. \& Contarini, P. (2005). In vitro activity of fluoroquinolones against Mycobacterium abscessus and Mycobacterium chelonae causing infectious keratitis after LASIK in Brazil. Cornea 24, 730-734.

Ito, H., Yoshida, H., Bogaki-Shonai, M., Niga, T., Hattori, H. \& Nakamura, S. (1994). Quinolone resistance mutations in the DNA gyrase gyrA and gyrB genes of Staphylococcus aureus. Antimicrob Agents Chemother 38, 2014-2023.

Leão, S. C., Viana-Niero, C., Matsumoto, C. K., Lima, K. V. B., Lopes, M. L., Palaci, M., Hadad, D. J., Vinhas, S., Duarte, R. S. \& other authors (2010). Epidemic of surgical-site infections by a single clone of rapidly growing mycobacteria in Brazil. Future Microbiol 5, 971980.

Leão, S. C., Tortoli, E., Euzéby, J. P. \& Garcia, M. J. (2011). Proposal that Mycobacterium massiliense and Mycobacterium bolletii be united and reclassified as Mycobacterium abscessus subsp. bolletii comb. nov., designation of Mycobacterium abscessus subsp. abscessus subsp. nov. and emended description of Mycobacterium abscessus. Int J Syst Evol Microbiol 61, 2311-2313.

Leysen, D. C., Haemers, A. \& Pattyn, S. R. (1989). Mycobacteria and the new quinolones. Antimicrob Agents Chemother 33, 1-5.

Li, X. Z., Zhang, L. \& Nikaido, H. (2004). Efflux pump-mediated intrinsic drug resistance in Mycobacterium smegmatis. Antimicrob Agents Chemother 48, 2415-2423.

Liu, J., Takiff, H. E. \& Nikaido, H. (1996). Active efflux of fluoroquinolones in Mycobacterium smegmatis mediated by LfrA, a multidrug efflux pump. J Bacteriol 178, 3791-3795.

Louw, G. E., Warren, R. M., Gey van Pittius, N. C., McEvoy, C. R. E., Van Helden, P. D. \& Victor, T. C. (2009). A balancing act: efflux/influx in mycobacterial drug resistance. Antimicrob Agents Chemother 53, 3181-3189.

Monego, F., Duarte, R. S. \& Biondo, A. W. (2011). gyrA and gyrB gene mutation in ciprofloxacin-resistant Mycobacterium massiliense clinical isolates from Southern Brazil. Microb Drug Resist http://dx.doi.org/ 10.1089/mdr.2011.0047 (Epub ahead of print)

Montero, C., Mateu, G., Rodriguez, R. \& Takiff, H. E. (2001). Intrinsic resistance of Mycobacterium smegmatis to fluoroquinolones may be influenced by new pentapeptide protein MfpA. Antimicrob Agents Chemother 45, 3387-3392.

Otto, T. D., Vasconcellos, E. A., Gomes, L. H. F., Moreira, A. S., Degrave, W. M., Mendonça-Lima, L. \& Alves-Ferreira, M. (2008). ChromaPipe: a pipeline for analysis, quality control and management for a DNA sequencing facility. Genet Mol Res 7, 861-871.

Pan, X. S., Ambler, J., Mehtar, S. \& Fisher, L. M. (1996). Involvement of topoisomerase IV and DNA gyrase as ciprofloxacin targets in Streptococcus pneumoniae. Antimicrob Agents Chemother 40, 23212326.

Phillips, M. S. \& von Reyn, C. F. (2001). Nosocomial infections due to nontuberculous mycobacteria. Clin Infect Dis 33, 1363-1374.

Reddy, A. K., Garg, P., Babu, K. H., Gopinathan, U. \& Sharma, S. (2010). In vitro antibiotic susceptibility of rapidly growing 
nontuberculous mycobacteria isolated from patients with microbial keratitis. Curr Eye Res 35, 225-229.

Revel, V., Cambau, E., Jarlier, V. \& Sougakoff, W. (1994). Characterization of mutations in Mycobacterium smegmatis involved in resistance to fluoroquinolones. Antimicrob Agents Chemother 38, 1991-1996.

Sampaio, J. L. (2010). Prokaryotic taxonomy rules and nomenclature changes in the Mycobacterium chelonae-abscessus group. Future Microbiol 5, 1457.

Scoper, S. V. (2008). Review of third-and fourth-generation fluoroquinolones in ophthalmology: in-vitro and in-vivo efficacy. Adv Ther 25, 979-994.

Stephan, J., Mailaender, C., Etienne, G., Daffé, M. \& Niederweis, M. (2004). Multidrug resistance of a porin deletion mutant of Mycobacterium smegmatis. Antimicrob Agents Chemother 48, 41634170.

Stroman, D. W., Dajcs, J. J., Cupp, G. A. \& Schlech, B. A. (2005). In vitro and in vivo potency of moxifloxacin and moxifloxacin ophthalmic solution $0.5 \%$, a new topical fluoroquinolone. Surv Ophthalmol 50 (Suppl. 1), S16-S31.

Svetlíková, Z., Skovierová, H., Niederweis, M., Gaillard, J. L., McDonnell, G. \& Jackson, M. (2009). Role of porins in the susceptibility of Mycobacterium smegmatis and Mycobacterium chelonae to aldehyde-based disinfectants and drugs. Antimicrob Agents Chemother 53, 4015-4018.

Takiff, H. E., Salazar, L., Guerrero, C., Philipp, W., Huang, W. M., Kreiswirth, B., Cole, S. T., Jacobs, W. R., Jr \& Telenti, A. (1994). Cloning and nucleotide sequence of Mycobacterium tuberculosis gyrA and $g y r B$ genes and detection of quinolone resistance mutations. Antimicrob Agents Chemother 38, 773-780.
Viana-Niero, C., Lima, K. V. B., Lopes, M. L., Rabello, M. C. S., Marsola, L. R., Brilhante, V. C. R., Durham, A. M. \& Leão, S. C. (2008). Molecular characterization of Mycobacterium massiliense and Mycobacterium bolletii in isolates collected from outbreaks of infections after laparoscopic surgeries and cosmetic procedures. J Clin Microbiol 46, 850-855.

Von Groll, A., Martin, A., Jureen, P., Hoffner, S., Vandamme, P., Portaels, F., Palomino, J. C. \& da Silva, P. A. (2009). Fluoroquinolone resistance in Mycobacterium tuberculosis and mutations in $g y r A$ and gyrB. Antimicrob Agents Chemother 53, 4498-4500.

Wallace, R. J., Jr, Brown, B. A. \& Griffith, D. E. (1998). Nosocomial outbreaks/pseudo-outbreaks caused by nontuberculous mycobacteria. Annu Rev Microbiol 52, 453-490.

Wang, J. Y., Lee, L. N., Lai, H. C., Wang, S. K., Jan, I. S., Yu, C. J., Hsueh, P. R. \& Yang, P. C. (2007). Fluoroquinolone resistance in Mycobacterium tuberculosis isolates: associated genetic mutations and relationship to antimicrobial exposure. J Antimicrob Chemother 59, 860-865.

Wolfson, J. S. \& Hooper, D. C. (1985). The fluoroquinolones: structures, mechanisms of action and resistance, and spectra of activity in vitro. Antimicrob Agents Chemother 28, 581-586.

Yew, W. W., Piddock, L. J. V., Li, M. S. K., Lyon, D., Chan, C. Y. \& Cheng, A. F. B. (1994). In-vitro activity of quinolones and macrolides against mycobacteria. J Antimicrob Chemother 34, 343-351.

Yoshida, H., Bogaki, M., Nakamura, M. \& Nakamura, S. (1990). Quinolone resistance-determining region in the DNA gyrase $g y r A$ gene of Escherichia coli. Antimicrob Agents Chemother 34, 1271-1272.

Yoshida, H., Bogaki, M., Nakamura, M., Yamanaka, L. M. \& Nakamura, S. (1991). Quinolone resistance-determining region in the DNA gyrase gyrB gene of Escherichia coli. Antimicrob Agents Chemother 35, 1647-1650. 